\title{
Parameter Sensitivity and Dependency Analysis for the WECC Dynamic Composite Load Model
}

\author{
Kaiqing Zhang, Siming Guo, Hao Zhu \\ Department of Electrical and Computer Engineering \\ University of Illinois at Urbana-Champaign, Urbana, IL, USA, 61801 \\ \{kzhang66, sguo6, haozhu\}@illinois.edu
}

\begin{abstract}
An accurate dynamic load model plays a crucial role in the analysis of power system transient stability. The WECC dynamic composite load model (CMPLDW) has been developed recently to better represent faultinduced delayed-voltage-recovery (FIDVR) events, which are of increasing concern to electric utilities. To facilitate the understanding of the CMPLDW, it is worth studying the effect of parameters that describe the model structure on its dynamic response. In this paper, we show that 1) some parameters have very minimal sensitivities under certain FIDVR events; 2) sensitivities of certain parameters are strongly dependent on the temporal profile of given fault, such as its minimum fault voltage or recovery time; and 3) some parameters share similar sensitivity patterns and thus the change of their values may complement each other. These observations are essential for further developing enhanced measurement-based dynamic load modeling approaches by tackling the parameter identifiability issues pointed out in the present work.
\end{abstract}

\section{Introduction}

It is essential to develop accurate dynamic load models for transient stability analysis and operation of power systems $[1,2]$. Nonetheless, the modeling has long been a challenging task due to the inherent uncertainty, complexity, and diversity of power system loads [3]. With the development of digital fault recorders, the measurement-based load modeling approach has become increasingly popular [4-9]. In this approach, the parameters describing a given model structure need to be determined by fitting field measurements during system disturbances. However, not all the parameters are identifiable using only field measurements according to some previous work [5-8], especially for complex model structures with large numbers of parameters. Hence the analysis of parameter sensitivity serves as the base for measurement-based load modeling.
There are several dynamic load model structures generally used for measurement-based load modeling. One popular choice consists of a static constant impedance-current-power (ZIP) component and a dynamic induction motor (IM) component. This ZIP+IM model has been widely used due to its simple structure [4-7]. In addition, the complex load model (CLOD) developed by PSS/E, which has been preliminarily investigated in [8], has several more components including two IMs with different torque-speed and current-speed curves. Although these load models are effective in representing load dynamic behavior during most short-duration faults, they fail to model the socalled fault-induced delayed-voltage-recovery (FIDVR) events; see $[10,11]$. Slow voltage recovery after lowvoltage faults is mainly caused by the stalling of lowinertia single-phase IMs. It is of increasing concern to utilities because of the resultant loss of voltage control and potential cascading effects. To model FIDVR events, the WECC composite load model (CMPLDW) has been developed [9], which includes a single-phase IM component among other enhancements characterized by 121 parameters. To the best of our knowledge, very few studies have been conducted to understand the identifiability of these parameters.

In general, the identifiability issues of load models has two causes [6]: the insensitivity of some parameters and the dependency among several parameters. Specifically, if the simulation results are completely insensitive to a parameter, then its value will not matter at all. Additionally, if the effects made by several parameters can always be complemented by each other, then different combinations of these parameter values will generate indistinguishable output measurements, based on which no unique parameter estimation can be obtained [12]. To investigate these causes, we perform trajectory sensitivity analysis to determine which parameters are sensitive. Based on this, we demonstrate dependency between pairs of parameters. To further quantify the dependency, we also perform singular value decomposition (SVD) on the sensitivities. 


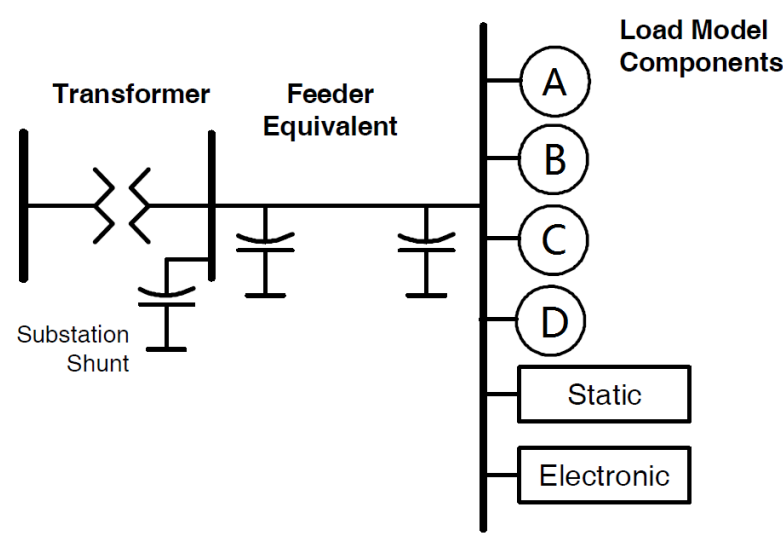

Figure 1: A schematic of the WECC CMPLDW composite load model [10].

This paper is organized as follows. Section 2 introduces the WECC CMPLDW and other background for the sensitivity analysis. Sections 3 and 4 present the results of the sensitivity and dependency analysis, respectively. Concluding remarks are provided and future work is addressed in Section 5.

\section{Background and objective}

This paper is focused on the WECC CMPLDW model and the modeling of FIDVR events. The structure of the model is illustrated in Figure 1, consisting of a substation transformer model, a feeder equivalent model, and six load model components [9]. The load components include three 3-phase motors, one singlephase motor, one static ZIP load, and one electronic load, all connected in parallel. The 3-phase motors are henceforth named motor $\mathrm{A}, \mathrm{B}$, and $\mathrm{C}$, and the singlephase motor, which is used to model an air conditioner compressor, is named motor D. Compared to the simple ZIP+IM model of only 13 parameters [5], the WECC CMPLDW has a more extensive list of parameters used to describe its static and dynamic behaviors. A representative CMPLDW model has in total 121 parameters with example values as listed in Table 1; see more details in [9]. These parameters, denoted as the vector $\boldsymbol{\theta}$, can be categorized to represent the following:

- substation and feeder (e.g., transformer reactance and feeder equivalent impedance);

- load model components (e.g., reactance and inertia of a motor, or the ZIP coefficients of static load);

- the fraction for each load component (i.e., Fel, FmA, etc.).

As shown in [4-7], dynamic load modeling is generally challenged by the unidentifiability of parameters that describe the model structure. The unidentifiability has two primary causes: the insensitivity of some parameters and the dependency among several parameters [6]. It is difficult to identify these parameters accurately because different values of insensitive parameters or various combinations of dependent parameters may result in a similar output response.

To facilitate the understanding of the model's identifiability, trajectory sensitivity analysis is performed to characterize each parameter. Specifically, the sensitivity of the system dynamic response to the changes of each parameter will be evaluated [13]. For complex load models such as the CMPLDW, it is difficult to develop their mathematical state-space representations to evaluate the trajectory sensitivity [4]. Hence, the finite-difference derivative approximation is employed to numerically evaluate the sensitivity [13]:

$$
\begin{aligned}
J_{i}(t): & =\left.\frac{\partial f(t ; \boldsymbol{\theta})}{\partial \theta_{i}}\right|_{\boldsymbol{\theta}=\boldsymbol{\theta}_{0}} \\
& \approx \frac{f\left(t ; \boldsymbol{\theta}_{0}+\epsilon \mathbf{e}_{i}\right)-f\left(t ; \boldsymbol{\theta}_{0}-\epsilon \mathbf{e}_{i}\right)}{2 \epsilon}
\end{aligned}
$$

where $\boldsymbol{\theta}_{0}$ is the vector of nominal parameter values, $\mathbf{e}_{i}$ is the Kronecker vector with all entries zero except for the $i$-th entry equal to 1 , and the perturbation coefficient $\epsilon$ is a small positive value. The dynamic system output $f(t)$ under different parameter settings can be obtained by numerical simulation. Hence, the resultant sensitivity $J_{i}(t)$ will consist of discrete-time samples throughout the fault event. Concatenating the samples into a vector $\mathbf{J}_{i}$ of length $T$ for each parameter, we can use it as the feature vector to characterize the effects of changing $\theta_{i}$ in the dynamic response. Note that perturbations in both active and reactive power outputs are included in the vector $\mathbf{J}_{i}$. Except for the parameters marked in italics in Table 1, most other parameters will be analyzed to see if they are insensitive or interdependent.

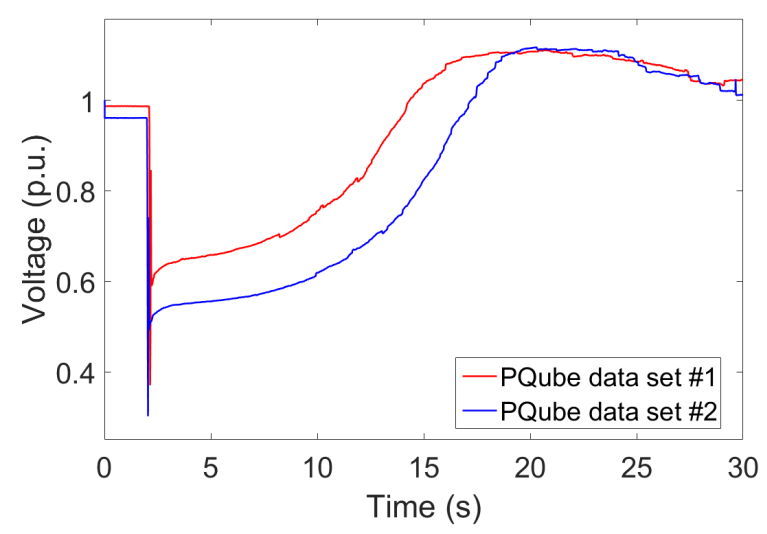

Figure 2: Two voltage profiles recorded by PQube devices during FIDVR events. 
Table 1: List of WECC CMPLDW Parameters with Example Values. Parameters in italics remain fixed by convention [10], and the 15 parameters in bold have the highest sensitivity.

\begin{tabular}{|c|c|c|c|c|c|c|c|c|c|c|c|}
\hline \multicolumn{2}{|c|}{ Feeder } & \multicolumn{2}{|c|}{ Static Load } & \multicolumn{2}{|c|}{ Motor A } & \multicolumn{2}{|c|}{ Motor B } & \multicolumn{2}{|c|}{ Motor C } & \multicolumn{2}{|c|}{ Motor D } \\
\hline Bss & 0 & Pfs & -0.99 & FmA & 0.167 & FmB & 0.167 & FmC & 0.167 & FmD & 0.167 \\
\hline Rfdr & 0.04 & P1e & 2 & MtypA & 3 & MtypB & 3 & MtypC & 3 & MtypD & 1 \\
\hline Xfdr & 0.05 & P1c & 0.54546 & LFmA & 0.7 & LFmB & 0.8 & LFmC & 0.8 & LFmD & 1 \\
\hline $\mathrm{Fb}$ & 0.75 & $\mathrm{P} 2 \mathrm{e}$ & 1 & RsA & 0.04 & RsB & 0.03 & $\mathrm{RsC}$ & 0.03 & CompPFD & 0.97 \\
\hline Xxf & 0.08 & $\mathrm{P} 2 \mathrm{c}$ & 0.45454 & LsA & 1.8 & LsB & 1.8 & LsC & 1.8 & VstallD & 0.6 \\
\hline Tfixhs & 1 & Pfrq & -1 & LpA & 0.1 & LpB & 0.16 & $\mathrm{LpC}$ & 0.16 & RstallD & 0.1 \\
\hline Tfixls & 1 & Q1e & 2 & LppA & 0.083 & LppB & 0.12 & LppC & 0.12 & XstallD & 0.1 \\
\hline$L T C$ & 1 & Q1c & -0.5 & TpoA & 0.092 & ТроB & 0.1 & TpoC & 0.1 & TstallD & 0.02 \\
\hline Tmin & 0.9 & Q2e & 1 & TppoA & 0.002 & TppoB & 0.0026 & TppoC & 0.0026 & FrstD & 0 \\
\hline Tmax & 1.1 & Q2c & 1.5 & HA & 0.05 & $\mathrm{HB}$ & 1 & $\mathrm{HC}$ & 0.1 & VrstD & 0.9 \\
\hline step & 0.00625 & Qfrq & -1 & EtrqA & 0 & EtrqB & 2 & EtrqC & 2 & TrstD & 0.4 \\
\hline$V m i n$ & 1 & & & Vtr1A & 0.75 & Vtr1B & 0.5 & Vtr1C & 0.5 & FuvrD & 0.17 \\
\hline Vmax & 1.02 & \multicolumn{2}{|c|}{ Electronic Load } & TtrlA & $\infty$ & $\mathrm{Ttr} 1 \mathrm{~B}$ & 0.02 & $\operatorname{Ttr} 1 \mathrm{C}$ & 0.02 & Vtr1D & 0.65 \\
\hline Tdel & 30 & Fel & 0.167 & Ftr1A & 0.2 & Ftr1B & 0.2 & Ftr1C & 0.2 & Ttr1D & 0.02 \\
\hline Tdelstep & 5 & Pfel & 1 & Vrc1A & 0.9 & Vrc1B & 0.65 & Vrc1C & 0.65 & Vtr2D & 0.9 \\
\hline Rcmp & 0 & Vd1 & 0.75 & $\operatorname{TrclA}$ & $\infty$ & $\operatorname{Trc} 1 \mathrm{~B}$ & 0.6 & $\operatorname{Trc} 1 \mathrm{C}$ & 0.6 & $\operatorname{Ttr} 2 \mathrm{D}$ & 5 \\
\hline Хстр & 0 & $\mathrm{Vd} 2$ & 0.65 & $\mathrm{Vtr} 2 \mathrm{~A}$ & 0.5 & Vtr2B & 0.7 & $\mathrm{Vtr} 2 \mathrm{C}$ & 0.7 & Vc1offD & 0.4 \\
\hline \multirow[t]{7}{*}{ Mbase } & 0 & Frcel & 0.25 & $\operatorname{Ttr2A}$ & 0.02 & $\operatorname{Ttr} 2 \mathrm{~B}$ & 0.02 & $\operatorname{Ttr} 2 \mathrm{C}$ & 0.02 & Vc2offD & 0.4 \\
\hline & & & & Ftr2A & 0.47 & Ftr2B & 0.3 & Ftr2C & 0.3 & Vc1onD & 0.45 \\
\hline & & & & Vrc2A & 0.639 & $\operatorname{Vrc} 2 \mathrm{~B}$ & 0.85 & $\operatorname{Vrc} 2 \mathrm{C}$ & 0.85 & Vc2onD & 0.45 \\
\hline & & & & $\operatorname{Trc} 2 \mathrm{~A}$ & 0.73 & $\operatorname{Tr} c 2 B$ & $\infty$ & $\operatorname{Trc} 2 C$ & $\infty$ & TthD & 30 \\
\hline & & & & & & & & & & Th1tD & 0.3 \\
\hline & & & & & & & & & & $\mathrm{Th} 2 \mathrm{tD}$ & 2.05 \\
\hline & & & & & & & & & & TvD & 0.025 \\
\hline
\end{tabular}

The event measurements are taken from real datasets collected by a southern US utility company. They are recorded using PQube devices [14] during three summer months in 2012. Each event includes the voltage profile and the corresponding real and reactive power consumption, all with cycle-level sampling rate (around $60 \mathrm{~Hz}$ ). Two FIDVR events have been identified from the data. As plotted in Figure 2, the voltage profile of FIDVR takes a long time, usually several seconds, to recover from the voltage drop caused by a certain disturbance. This phenomenon generally results from the high penetration of single-phase induction motors in power systems, which can barely be captured by the traditional ZIP+IM model structure. We thus mainly focus on addressing FIDVR type faults in the ensuing analysis.

\section{Parameter trajectory sensitivity analysis}

Main results from the parameters' trajectory sensitivity under FIDVR events are illustrated in this section. Given a fault voltage disturbance, we first show that some parameters have very minimal (even absolutely zero) sensitivity. Interestingly, we make further observations that the sensitivity is strongly related to the shape of the faulted voltage, which originates from the thresholding nonlinearity of the WECC CMPLDW.

\subsection{Insensitive parameters}

Using the sensitivity vectors $\left\{\mathbf{J}_{i}\right\}$, we can first identify the parameters that the load model output is insensitive to. Figure 3 plots the sorted $\left\|\mathbf{J}_{i}\right\|_{2}$ values and their logarithmic values for all CMPLDW parameters under the voltage input in dataset \#1. Clearly, almost half the parameters have very minimal, or even zero, trajectory sensitivity, which means that changing 


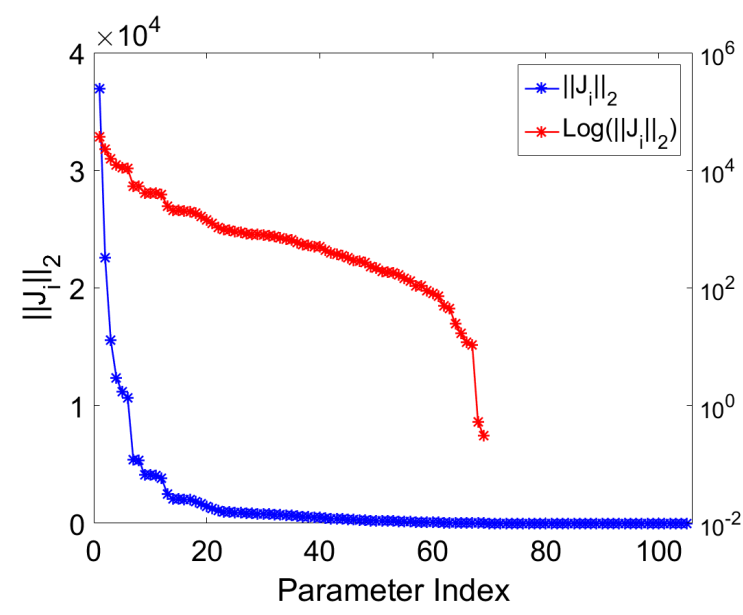

Figure 3: The 2-norm and its logarithm of the trajectory sensitivity of each parameter from the test on PQube data set \#1, sorted in descending order.

the value of several CMPLDW parameters could result in negligible output perturbations. Hence, we choose a predetermined threshold $r_{t h}>0$ and only select the parameters with $\left\|\mathbf{J}_{i}\right\|_{2} \geq r_{t h}$ for the ensuing analysis. For the $N$ parameters selected, their corresponding feature vectors are stacked into the $T \times N$ matrix $\mathbf{J}$, which is approximately the Jacobian matrix for the measurement function $\mathbf{f}(\boldsymbol{\theta})$ at $\boldsymbol{\theta}=\boldsymbol{\theta}_{0}$. The most significantly sensitive parameters are marked as bold in Table 1. Particularly, by setting the threshold $r_{t h}$ as $1 \%$ of the nominal output norm $\left\|\mathbf{J}_{i}\right\|_{2}$, a total of $N=70$ parameters are selected to form the resultant Jacobian $\mathbf{J}$.

Some of the absolutely insensitive parameters are related to the tripping behavior of the load components, which may not be activated under the given input voltage profile. For example, parameters $\mathrm{Vd} 1$ and $\mathrm{Vd} 2$ are used to set the voltage levels for tripping the electronic load. Slightly varying their values will not cause any change of the system response if the lowest voltage input is significantly higher than the initial tripping levels specified in $\mathrm{Vd} 1$ and $\mathrm{Vd} 2$. These examples reveal the thresholding nonlinearity of the CMPLDW model. Therefore, the selection of insensitive parameters depends on both the initial parameter setting $\boldsymbol{\theta}_{0}$ and the input voltage profile.

\subsection{Impact of input fault voltage profile}

Building on previous analysis, we investigate the impact of the input voltage profile on the parameter sensitivity. We first designate PQube data set \#1 in Figure 2 as our base case. We then modify the base case profile to emulate faults of varying severity and recovery time. Specifically, we scale the profile vertically to vary the minimum fault voltage, while maintaining the pre-fault voltage. We scale the profile horizontally to vary the recovery time. These are both illustrated in Figure 4.

The parameter sensitivities are then calculated for

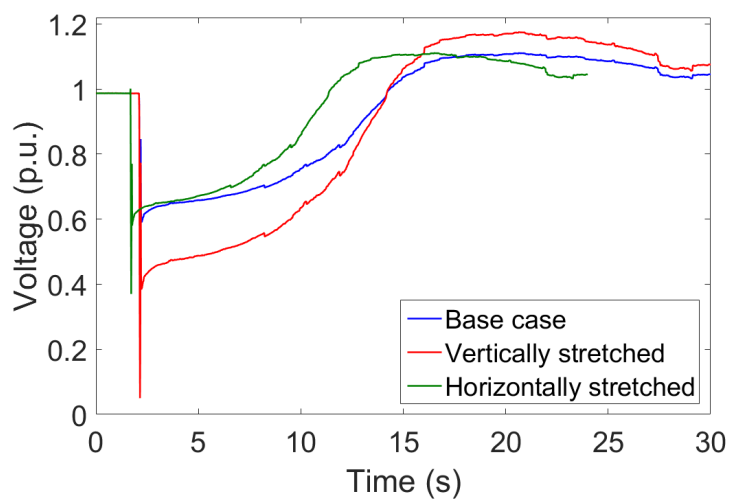

Figure 4: Illustration of vertical and horizontal stretching of the voltage profile.

each voltage profile and plotted in Figure 5 and Figure 6 on a log scale. From Figure 5 we can see that the parameter sensitivities do not remain constant. There is a cyan colored line which has relatively low sensitivity between 0 and 0.5 p.u., peaks at 0.6 p.u., then disappears for profiles with higher minimum voltage. This line corresponds to the parameter VstallD, the stall voltage for motor D. In the base case, the stall voltage is set to 0.6 p.u.. When the minimum fault voltage does not drop below 0.6 p.u., motor D will not stall, hence VstallD does not affect the trajectory at all $\left(\left\|\mathbf{J}_{i}\right\|_{2}=0\right.$ for VstallD). Since Figure 5 uses a log axis, those zero sensitivities cannot be plotted. On the other hand, when the minimum fault voltage is very close to 0.6 p.u., the sensitivity of VstallD exceeds the other parameters approximately one order of magnitude. This is because a small change in the value of VstallD will determine whether motor D stalls; this is thresholding nonlinearity. Finally, for faults where the voltage drops significantly below 0.6 p.u., motor D will definitely stall, so the trajectory again becomes very insensitive to VstallD. The reason the sensitivity is not exactly zero is merely because a more severe fault causes the voltage to drop faster, and hence causes motor D to stall a fraction of a cycle earlier.

We can also see that many parameters have a large peak in sensitivity when the minimum fault voltage is 0.7 p.u. and a few parameters have a peak at 0.5 p.u.. These can also be attributed to thresholding nonlinearity. Voltage trip levels Vtr1A, Vtr2B, Vtr2C, and Vtr1D, and voltage reconnection levels $\mathrm{Vrc} 1 \mathrm{~B}, \mathrm{Vrc} 1 \mathrm{C}$, and $\mathrm{Vrc} 2 \mathrm{~A}$ are all between 0.639 and 0.75 p.u.. Voltage trip levels Vtr1B, Vtr1C, and Vtr2A are all 0.5 p.u.. When the minimum fault voltage is near those values, not only those voltage thresholds, but also any associated time delays, such as trip delay times, become very sensitive. For 


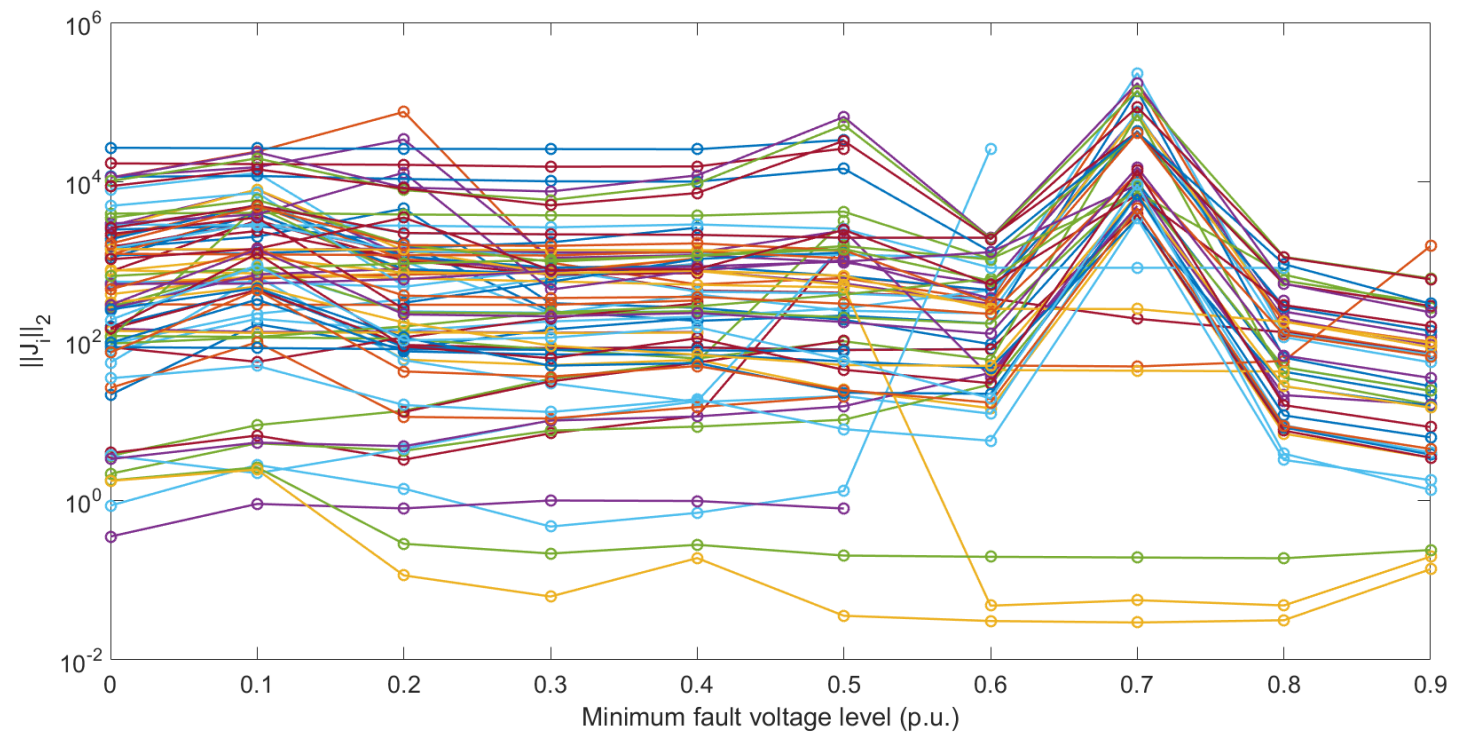

Figure 5: Parameter sensitivity under vertical stretching of the voltage profile. The base case minimum fault voltage level is $0.3703 \mathrm{pu}$ (see Figure 2).

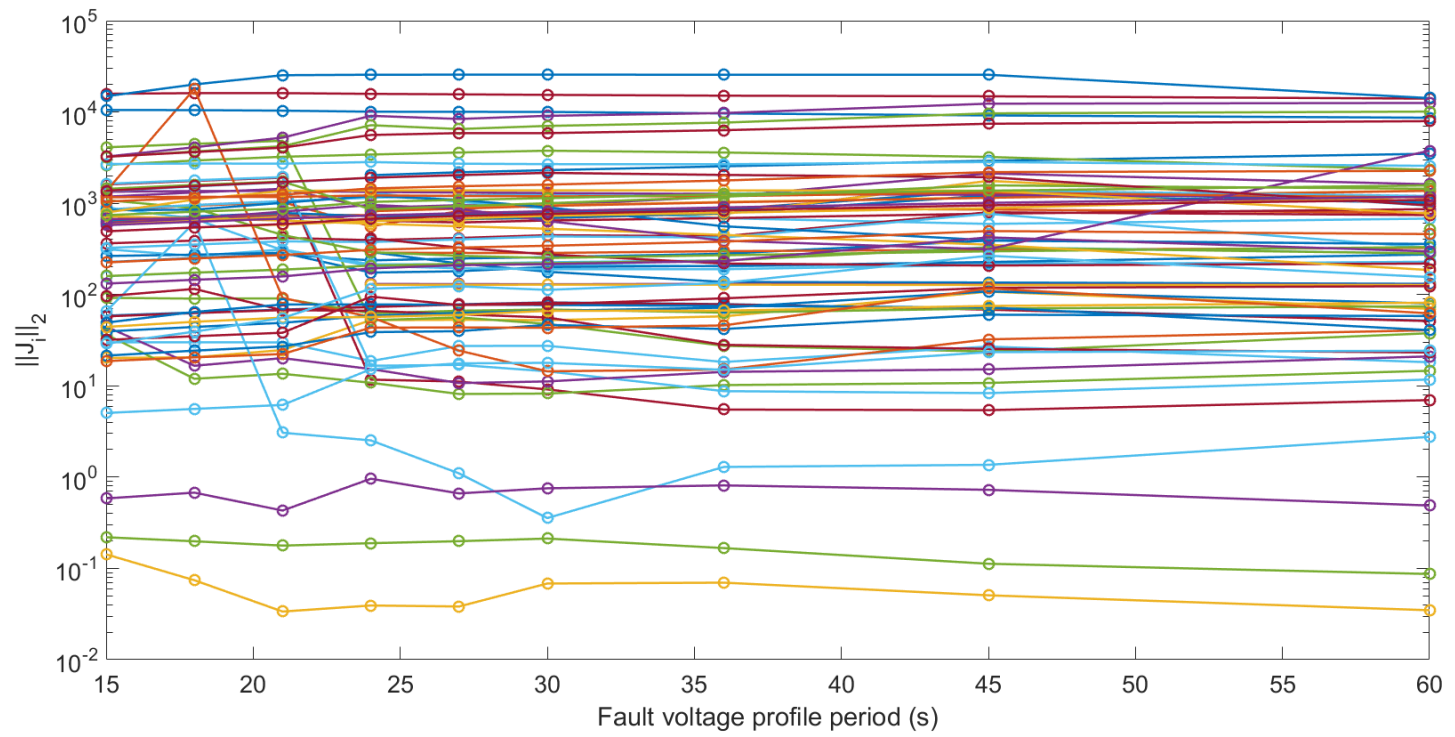

Figure 6: Parameter sensitivity under horizontal stretching of the voltage profile. The base case profile period is $30 \mathrm{~s}$ (see Figure 2).

example, motor B parameters Vtr1B and Ttr1B represent the under-voltage trip level and the trip delay time. If motor B does not trip for the given voltage input, varying Ttr1B will not affect the output dynamics either. The parameter sensitivities in Figure 6 are more uniform than those in Figure 5. This is because we are not affecting the minimum fault voltage. However, we still see some peaks when the profile period is reduced to around $20 \mathrm{~s}$, which is evidence that thresholding non-linearity is occuring for time thresholds, such as a reconnection time delay.

\section{Parameter dependency analysis}

In Section 3.1, we showed that the output response was insensitive to about half of the parameters, meaning those parameters cannot be observed with the given measurements. However, even among the parameters with high sensitivity, it may still be difficult to uniquely identify a parameter. This is due to parameter dependency: the effect of one parameter on the output response can be replicated by one or more other parameters. 


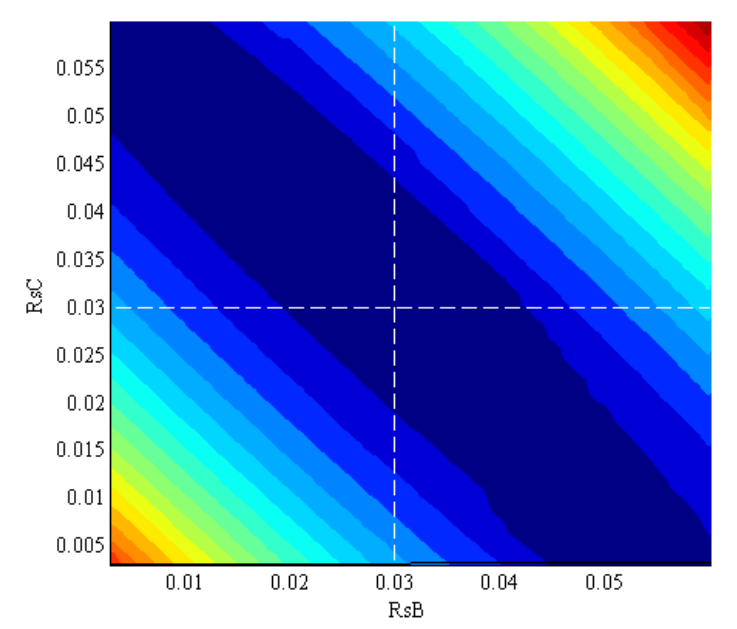

Figure 7: Contour of the MSE between the base case output and test cases for $\mathrm{RsB}$ and $\mathrm{RsC}$, the stator resistances of motors B and C.

\subsection{Examples of dependent parameters}

As an example, consider the stator resistance of motors B and C, RsB and RsC. Since the parameters of motors $\mathrm{B}$ and $\mathrm{C}$ are quite similar, we find that $\mathrm{RsB}$ and $\mathrm{RsC}$ are essentially indistinguishable from one another. In Figure 7, the default values of RsB and $\mathrm{RsC}$ (see Table 1) are indicated by the dashed white lines. The simulation result with RsB and RsC at their default values is the base case output. With all other parameters fixed, the values of $\mathrm{RsB}$ and $\mathrm{RsC}$ are then varied between $10 \%$ and $200 \%$ of their default value. The mean squared error (MSE) between the resulting outputs and the base case output are then calculated and contoured in the figure. From the figure, we can see that as long as RsB and RsC are varied proportionally, the MSE will stay in the dark blue region extending from the top-left to bottom-right corners. Thus, from a single set of output measurements, it is difficult to uniquely identify both RsB and RsC. Only if we pick one parameter's value can we then determine the other parameter.

While the dependence of this particular pair of parameters seems intuitive, there are other pairs of dependent parameters which have no simple explanation. Consider the following two parameters for the single-phase motor D: XstallD, the stall reactance in p.u., and Th2tD, the thermal protection trip completion temperature in p.u.. Both these parameters have quite high sensitivity: XstallD is ranked first, and Th2tD is 13th among all the parameters. These two parameters are also completely unrelated from a physical standpoint. However, in Figure 8, we can see that they exhibit interdependent behavior. We hypothesize that

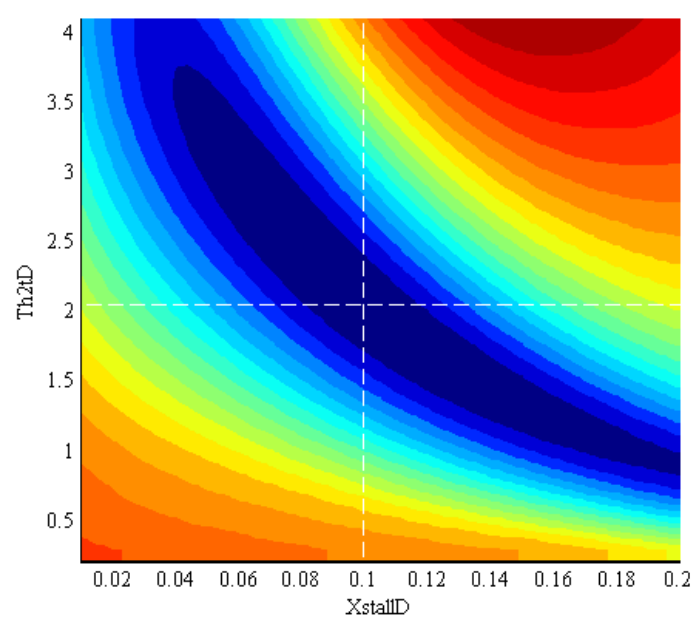

Figure 8: Contour of the MSE for two relatively sensitive parameters. XstallD and Th2tD are the stall reactance and the thermal protection trip completion temperature for motor $D$, respectively.

this is because for a larger XstallD, the stall current and hence the thermal losses would be lower, which would require a lower Th2tD to cause the same tripping behavior. Due to this interdependency, it would still be difficult to identify both parameters uniquely within the dark blue region.

Finally, we illustrate how thresholding nonlinearity impacts the parameter dependency by contouring VstallD and XstallD. VstallD is the stall voltage of motor D, which we also highlighted in Figure 5 and Section 3.2. This Figure 9 agrees with our analysis from

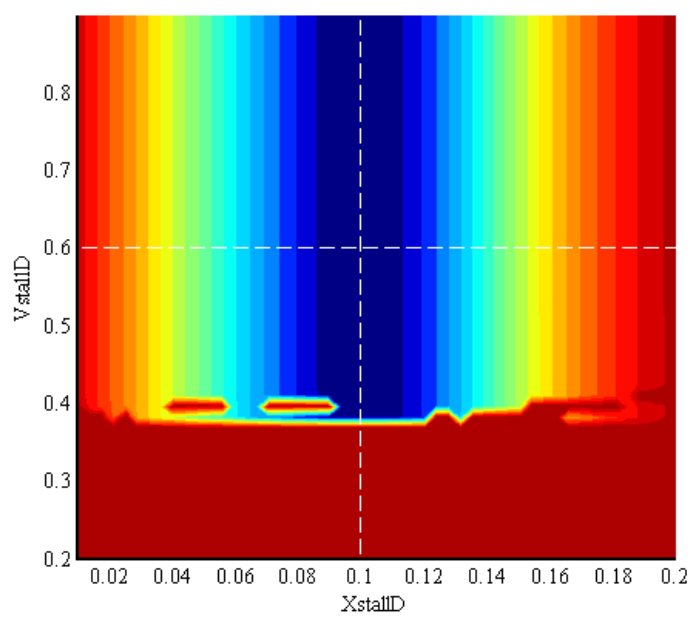

Figure 9: Contour of the MSE which highlights the effect of thresholding nonlinearity. XstallD, the stall reactance for motor $D$, is continuous, but VstallD, the stall voltage of motor $\mathrm{D}$, is discontinuous. 
Section 3.2. Note that we change the voltage trip level here, whereas Figure 5 changed the minimum fault voltage. When the stall voltage VstallD is set below the minimum fault voltage of 0.3703 p.u., neither its own value nor that of the stall reactance matters, which leads to the dark red area at the bottom of the contour. Above 0.3703 p.u., the vertical contour lines mean that VstallD does not matter. However, since VstallD is higher than the minimum fault voltage, the motor will stall, so XstallD does impact the simulation result.

\subsection{SVD-based dependency analysis}

As revealed in Section 4.1, there exist possible dependencies among parameters. To further quantify this, singular value decomposition [15] on matrix $\mathbf{J}$ is performed to find the most essential components. Additionally, since the length of each $\mathbf{J}_{i}$ is the total number of output samples, its value $T$ can be very large. This may pose a challenge when performing data analytics on the parameter features [16]. To remedy this, SVDbased analysis can also be used to reduce the dimension of the trajectory sensitivity.

To analyze the dependency of parameters with vastly different sensitivities, we first normalize each column $\mathbf{J}_{i}$ to be in the range $[0,1]$, to make the parameters comparable. With $T>>N$, the compact SVD of the Jacobian matrix $\mathbf{J}$ is given by

$$
\mathbf{J}=\mathbf{U} \cdot \boldsymbol{\Sigma} \cdot \mathbf{V}^{\mathrm{T}}
$$

where the $T \times N$ matrix $\mathbf{U}=\left[\mathbf{u}_{1}, \ldots, \mathbf{u}_{N}\right]$ consists of the $N$ orthonormal left-singular vectors satisfying $\mathbf{U}^{\mathrm{T}} \mathbf{U}=\mathbf{I}$; and similarly for the right-singular vectors in the $N \times N$ matrix $\mathbf{V}=\left[\mathbf{v}_{1}, \ldots, \mathbf{v}_{N}\right]$. The diagonal matrix $\boldsymbol{\Sigma}=\operatorname{diag}\left\{\sigma_{1}, \sigma_{2}, \cdots, \sigma_{N}\right\}$ contains the $N$ singular values ordered by $\sigma_{1} \geq \sigma_{2} \geq \cdots \geq$ $\sigma_{N} \geq 0$. Each column of (2) can be represented as $\mathbf{J}_{i}=\sum_{n=1}^{N}\left(\sigma_{n} v_{n, i}\right) \mathbf{u}_{n}$, where $v_{n, i}$ is the $i$-th entry of $\mathbf{v}_{n}$. Hence, each $\mathbf{u}_{n}$ can be viewed as a common principle component (PC) of all vectors $\left\{\mathbf{J}_{i}\right\}$.

Figure 10 plots all the $N$ singular values for the normalized Jacobian matrix. Clearly, the magnitude of $\sigma_{n}$ decreases rapidly when $n \leq 20$, implying the first few PCs are much more dominant in forming $\mathbf{J}_{i}$ compared to the rest. This further verifies the dependency of these parameters. Particularly, $\mathbf{J}_{i}$ can be well approximated using the first $L<N$ PCs, as given by $\mathbf{J}_{i} \approx \mathbf{J}_{i}^{L}:=\sum_{n=1}^{L}\left(\sigma_{n} v_{n, i}\right) \mathbf{u}_{n}$. In fact, the approximation error ratio using the first $L$ PCs can be quantified using [15]

$$
\eta_{L}:=\frac{\left\|\mathbf{J}-\mathbf{J}^{L}\right\|_{F}}{\|\mathbf{J}\|_{F}}=\frac{\sqrt{\sum_{n=L+1}^{N} \sigma_{n}^{2}}}{\sqrt{\sum_{n=1}^{N} \sigma_{n}^{2}}}
$$

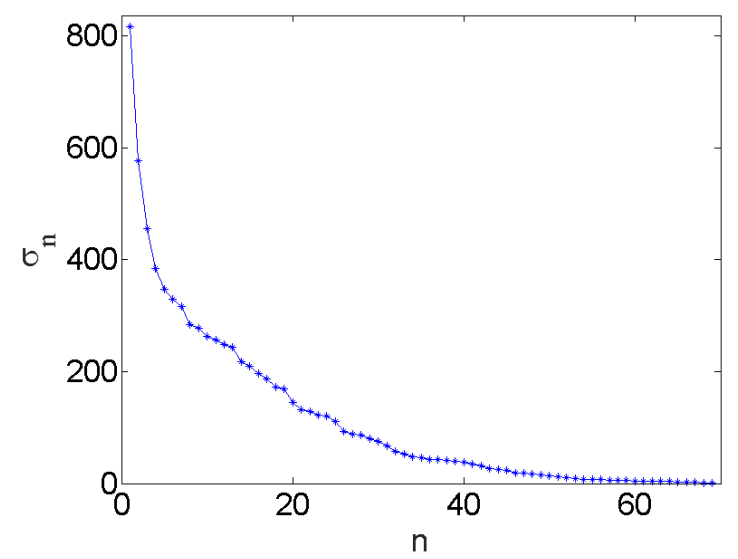

Figure 10: The singular values $\sigma_{n}$ of the normalized Jacobian matrix in descending order.

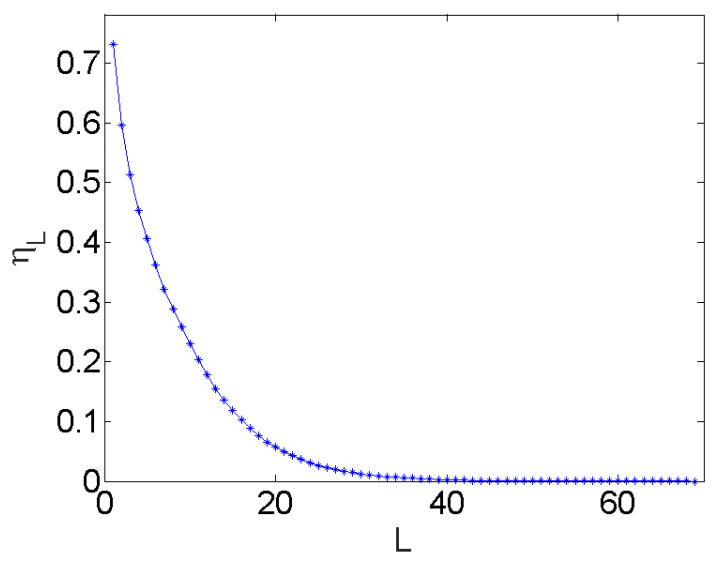

Figure 11: The approximation error ratio $\eta_{L}$ of the normalized Jacobian matrix using the first $L$ PCs.

where $\|\cdot\|_{F}$ denotes the matrix Frobenius norm. Figure 11 plots the approximation error ratio value versus the number of PCs $L$. To achieve $\eta_{L}<10 \%$ one can select $L=16$ PCs, while for $\eta_{L}<1 \%, L=35$ suffices. Hence, we can approximate each $\mathbf{J}_{i}$ of length $T$ using a much smaller number of PCs with excellent approximation accuracy. We will further explore this in our future work.

\section{Conclusions and future work}

This paper is focused on the identifiability issues of the CMPLDW load model developed by WECC. By performing sensitivity analysis on the CMPLDW model, we show that the output response has varying degrees of sensitivity to each parameter. Although the exact trajectory sensitivity is linked to the shape of the voltage 
profile, we can still make the general conclusion that for approximately half the parameters, the sensitivity varies across seven orders of magnitude. For the remaining parameters, the sensitivity is zero. This is primarily due to thresholding nonlinearity related to voltage trip levels and their associated time delays and recovery characteristics. Even among the sensitive parameters, however, we identify pairs of dependent parameters. Some are intuitive, but there are also pairs of physically unrelated parameters that behave similarly. To further quantify this dependency, we use SVD analysis to show that we can maintain $99 \%$ accuracy by considering only 35 principal components, and $90 \%$ accuracy by using only 16.

SVD-based analysis can also be used to reduce the dimension of the trajectory sensitivity. The next step will be to create an automated process to identify the sets of parameters which are interdependent. Based on that analysis, we can reduce the dimension of the parameter to be estimated, while still maintaining a high degree of accuracy.

\section{Acknowledgments}

The authors would like to thank the Power Systems Engineering Research Center (project S-60) and the Bonneville Power Administration (project TIP 357) for funding this work.

\section{References}

[1] M. Kent, W. Schmus, F. McCrackin, and L. Wheeler, "Dynamic modeling of loads in stability studies," IEEE Trans. Power Appar. Syst., vol. PAS-88, no. 5, pp. 756-763, 1969.

[2] J. A. De Leon and B. Kehrli, "The modeling requirements for short-term voltage stability studies," in Proc. IEEE PES Power Syst. Conf. Expo., Oct. 2006, pp. 582-588.

[3] I. A. Hiskens and J. Alseddiqui, "Sensitivity, approximation, and uncertainty in power system dynamic simulation," IEEE Trans. Power Syst., vol. 21, no. 4, pp. 1808-1820, 2006.

[4] J. Ma, D. Han, R. He, Z. Dong, and D. J. Hill, "Reducing identified parameters of measurementbased composite load model," IEEE Trans. Power Syst., vol. 23, no. 1, pp. 76-83, 2008.

[5] B.-K. Choi and H.-D. Chiang, "Multiple solutions and plateau phenomenon in measurement-based load model development," IEEE Trans. Power Syst., vol. 24, no. 2, pp. 824-831, 2009.

[6] S. Son, S.-H. Lee, D.-H. Choi, K.-B. Song, J.D. Park, Y.-H. Kwon, K. Hur, and J.-W. Park,
"Improvement of composite load modeling based on parameter sensitivity and dependency analyses," IEEE Trans. Power Syst., vol. 29, no. 1, pp. 242-250, 2014.

[7] J.-K. Kim, K. An, and J. Ma, "Fast and reliable estimation of composite load model parameters using analytical similarity of parameter sensitivity," IEEE Trans. Power Syst., vol. 31, no. 1, pp. 663-671, 2016.

[8] S. Guo and T. J. Overbye, "Parameter estimation of a complex load model using phasor measurements," in Proc. Power \& Energy Conf. at Illinois, Feb. 2012, pp. 1-6.

[9] D. Kosterev, A. Meklin, J. Undrill, B. Lesieutre, W. Price, D. Chassin, R. Bravo, and S. Yang, "Load modeling in power system studies: WECC progress update," in Proc. IEEE PES Gen. Meet., Jul. 2008.

[10] A. Borden and B. Lesieutre, "Model validation: FIDVR event," University of Wisconsin-Madison, Madison, WI, Tech. Rep., 2009.

[11] I. Widjaja, D. Latulipe, D. D’Aquila, G. Ng, and K. Swe, "SS-38 load modeling working group progress report," NERC, Tech. Rep., 2016.

[12] K. Zhang, H. Zhu, and S. Guo, "Dependency analysis and improved parameter estimation for dynamic composite load modeling," IEEE Trans. Power Syst., 2016, (under revision).

[13] I. Hiskens and M. Pai, "Trajectory sensitivity analysis of hybrid systems," IEEE Trans. Circuits Syst., vol. 47, no. 2, pp. 204-220, 2000.

[14] PQube Installation and User Manual, Power Standards Lab., 2015.

[15] G. H. Golub and C. F. Van Loan, Matrix Computations, 3rd ed. The Johns Hopkins University Press, 1996.

[16] M. Steinbach, L. Ertoz, and V. Kumar, "The challenges of clustering high dimensional data," in New Vistas in Statistical Physics, 2004, pp. 273309. 